\title{
Severe peritonitis after successful endoscopic ultrasound-guided gallbladder drainage for acute gangrenous cholecystitis
}

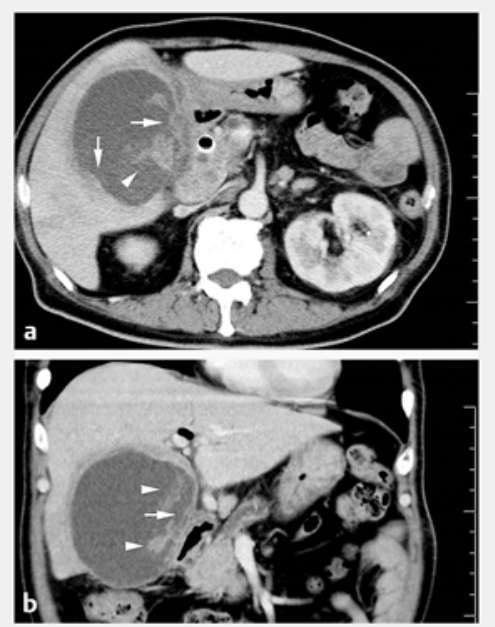

- Fig. 1 Contrast-enhanced computed tomography showed a markedly distended gallbladder with a severely irregular wall (arrows) and intraluminal membranes (arrowheads) indicating gangrenous cholecystitis. a Axial view. b Coronal view.

A 64-year-old man with unresectable pancreatic cancer, who had previously undergone placement of a fully covered self-expandable metallic stent (FCSEMS) in the bile duct, experienced acute cholecystitis.

Contrast-enhanced computed tomography revealed a markedly distended gallbladder with a severely irregular wall and intraluminal membranes indicating gangrenous cholecystitis (> Fig.1, - Video 1) [1]. As percutaneous gallbladder aspiration without tube placement was ineffective, endoscopic ultrasoundguided transluminal gallbladder drainage (EUS-GBD) was performed (> Fig.2, - Video 1).

Following puncture with a 19-gauge needle and dilation with a 7-Fr bougie catheter, an FCSEMS (WallFlex, 10×60 mm; Boston Scientific Japan K.K., Tokyo, Japan) was deployed at the puncture site, bridging the gallbladder and stomach. In addition, a 7-Fr double-pigtail plastic

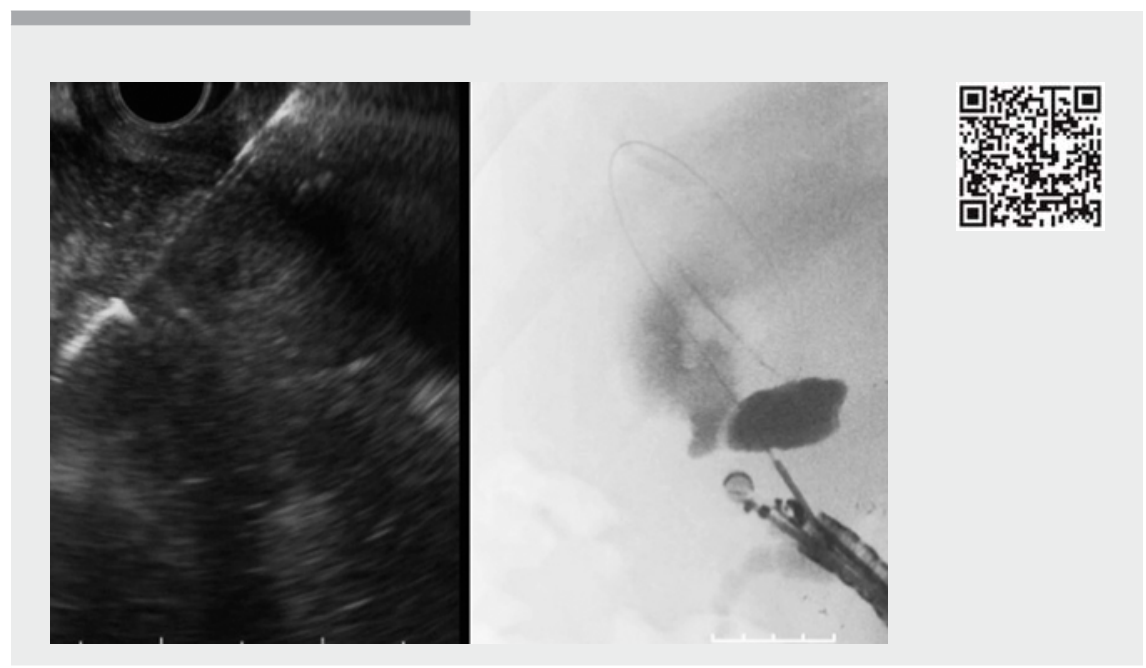

$\square$ Video 1 Endoscopic ultrasound-guided transluminal gallbladder drainage.

stent was placed across the FCSEMS as an anchor to prevent stent migration. Despite the successful unimpeded endoscopic procedure, generalized peritonitis with a notable amount of ascites and pneumoperitoneum occurred the following day ( $>$ Fig. 3, \ Video 1).

Emergency surgery revealed the presence of $2500 \mathrm{~mL}$ of dark-colored ascites, with a dirty appearance, although the stents appropriately remained astride the gallbladder and stomach. Cholecystectomy and closure of the gastric hole where the stents had been placed were performed, resulting in a remarkable improvement in the patient's condition. We speculatively concluded that bile and gastric fluid would continuously leak along the FCSEMS because the gangrenous gallbladder wall was too loose and fragile to sufficiently tighten the stent.

Several studies [2,3] have reported EUS-GBD as an alternative to percutaneous transhepatic gallbladder drainage (PTGBD) for acute cholecystitis, especially in cases with advanced malignancy. However, severe bile leakage may be more frequent following EUS-GBD than PTGBD procedures because of nonadhesion between the gallbladder and gastrointestinal tract, whereas the PTBGD route can involve an intervening liver, which adheres to the gallbladder wall. Gangrenous cholecystitis might be a risk factor for severe bile leakage in gallbladder drainage; therefore, PTGBD would be preferable to EUS-GBD in such patients.

Endoscopy_UCTN_Code_TTT_1AS_2AG

Competing interests

None

The authors

Toji Murabayashi ${ }^{1}$, Yoshihide Kanno ${ }^{1}$, Kei Ito ${ }^{1}$, Shinsuke Koshita ${ }^{1}$, Takahisa Ogawa ${ }^{1}$, Masaya Oikawa², Yutaka Noda'

1 Department of Gastroenterology, Sendai City Medical Center, Sendai, Japan

2 Department of Surgery, Sendai City Medical Center, Sendai, Japan 

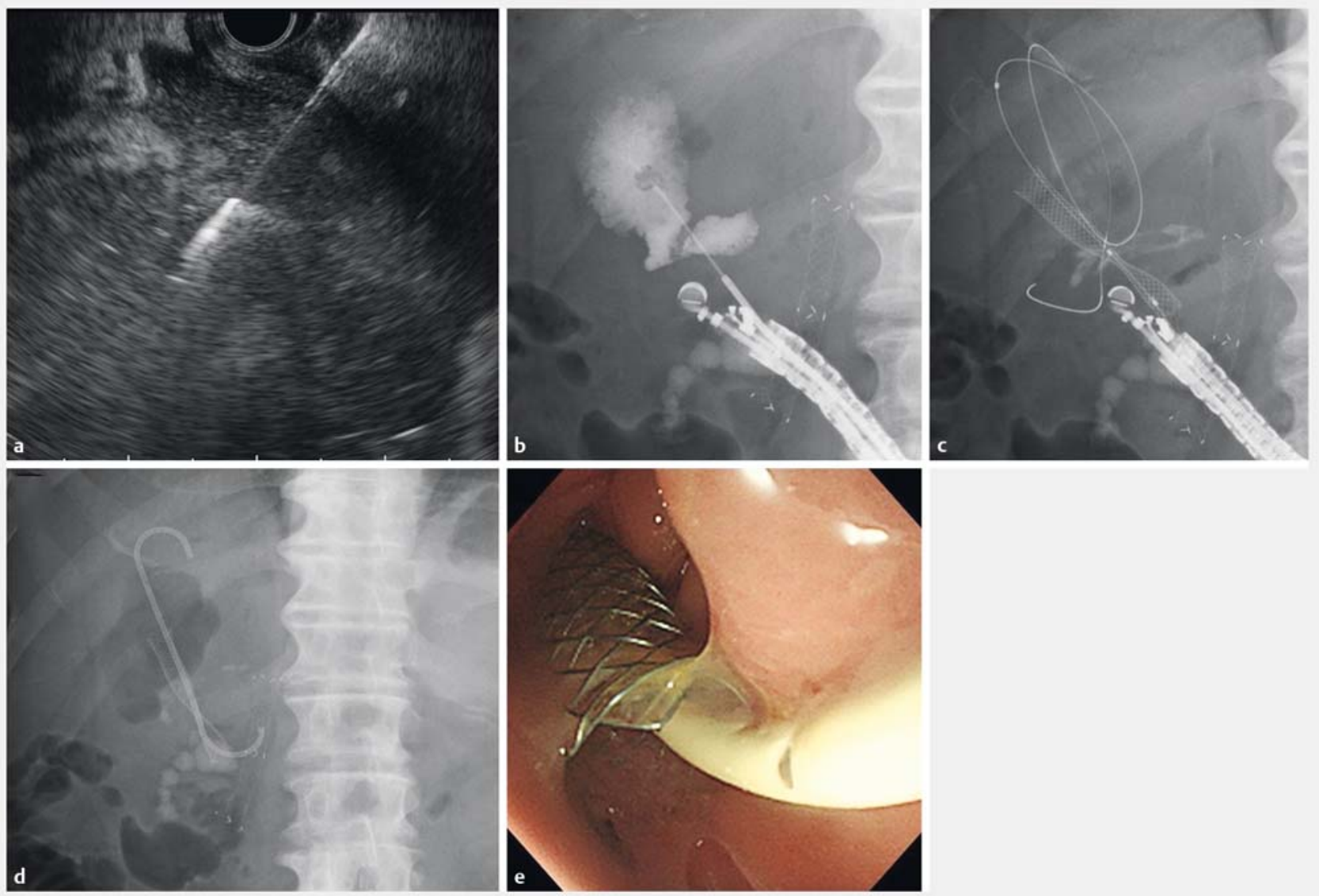

- Fig. 2 Endoscopic ultrasound (EUS)-guided transluminal gallbladder drainage. a The gallbladder was punctured with a 19-gauge needle via the antrum under EUS guidance. $\mathbf{b}$ Injection of contrast medium. c A fully covered self-expandable metallic stent (FCSEMS) was deployed at the puncture tract. d, e A double-pigtail plastic stent was deployed across the FCSEMS as an anchor.
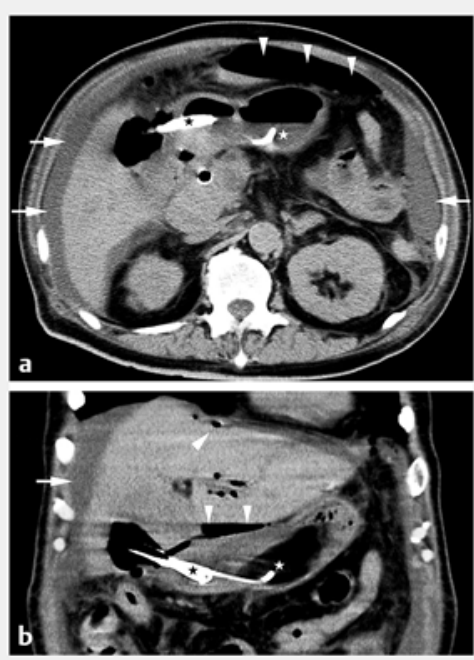

Fig. 3 Computed tomography showed a notable amount of ascites (arrows) and pneumoperitoneum (arrowheads). The stents (stars) remained bridging the stomach and gallbladder. a Axial view. b Coronal view.

\section{Corresponding author}

\section{Toji Murabayashi, MD}

Department of Gastroenterology, Sendai City Medical Center, 5-22-1, Tsurugaya, Miyagino-ku, Sendai 983-0824, Japan Fax: +81-22-2529431

murabayashi@openhp.or.jp

\section{References}

[1] Bennett GL, Rusinek H, Lisi V et al. CT findings in acute gangrenous cholecystitis. AJR 2002; 178: 275-281

[2] Jang JW, Lee SS, Song TJ et al. Endoscopic ultrasound-guided transmural and percutaneous transhepatic gallbladder drainage are comparable for acute cholecystitis. Gastroenterology 2012; 142: 805-811

[3] Choi JH, Kim HW, Lee JC et al. Percutaneous transhepatic versus EUS-guided gallbladder drainage for malignant cystic duct obstruction. Gastrointest Endosc 2017; 85: 357 364

\section{Bibliography}

DOI https://doi.org/10.1055/a-0591-2630

Published online: 9.4.2018

Endoscopy 2018; 50: 642-643

(c) Georg Thieme Verlag KG

Stuttgart · New York

ISSN 0013-726X

\section{CORRECTION}

Murabayashi T, Kanno Y, Ito K et al. Severe peritonitis after successful endoscopic ultrasound-guided gallbladder drainage for acute gangrenous cholecystitis.

Endoscopy 2018, 50:

doi:10.1055/a-0591-2630

In the above mentioned article the page numbers have been corrected. This was corrected in the online version on August 17, 2018. 